\title{
Inspección inteligente de líneas de alta tensión con drones
} Intelligent Inspection of Power Lines with Drones

Javier A. Meza-Mora ${ }^{1}$, Adolfo Gómez-Astúa², Alejandro Prado-Mora ${ }^{3}$

Meza-Mora, J.A; Gómez-Astúa, A; Prado-Mora, A. Inspección inteligente de líneas de alta tensión con drones. Tecnología en Marcha. Vol. 34, especial. ALTAE. Diciembre 2021. Pág 62-69.

doi) https://doi.org/10.18845/tm.v34i7.6014 


\title{
Palabras clave
}

Mantenimiento; líneas de transmisión; drones; inspección.

\section{Resumen}

Las líneas eléctricas recorren todo tipo de terrenos, incluso algunos no accesibles por tierra, por lo que este mantenimiento se lleva a cabo usando helicópteros y vehículos todo terreno, sin dejar de mencionar un peligro inherente para las personas que en tierra o escalando la infraestructura requieren muchas veces tener riesgosos movimientos que realizar en campo y en la misma infraestructura, lo que lo hace costoso, lento e ineficiente considerando que las líneas eléctricas se tienden por miles de kilómetros. El mantenimiento en líneas eléctricas de transmisión es un trabajo necesario para brindar un servicio de calidad, actualmente presenta ciertas limitaciones ya que es un trabajo riesgoso. Utilizando cámaras termográficas, lentes de zoom óptico, aeronaves no tripuladas podemos obtener la información necesaria sin necesidad de cortar la electricidad.

\section{Keywords}

Drone; power lines; electricity; transmission.

\begin{abstract}
Aerial inspection services for power line by drone, suitable for operations at low altitude, low speed and low moderate wind conditions, equipped with photography and filming equipment high resolution color for specific spans in transmission lines of the scope.
\end{abstract}

\section{Introducción}

GEOINN Geospatial Innovations ha puesto en marcha el servicio de inspección aérea para líneas de transmisión mediante Drones o Sistema de Aeronaves Pilotadas a Distancia (RPAS). Con el objetivo de incrementar la eficiencia en las labores de mantenimiento y alcanzar una mayor confiabilidad en el sistema de transmisión, GEOINN se encuentra brindando el servicio de inspección torre por torre con la línea energizada, y trabaja en la mejora continua de estos servicios para incrementar la usabilidad, así como la eficiencia del proceso y de la utilización misma de los datos en procesos inteligentes dentro de las mismas organizaciones.

Aspectos generales que se toman en cuenta:

- La condición de las cadenas de aisladores.

- La condición de los conductores, separadores, amortiguadores, cuellos y conexiones terminales.

- La condición del cable de guarda, incluyendo todos sus elementos en el punto de anclaje a la torre.

Este tipo de inspección consiste en hacer una evaluación minuciosa en toda la línea y sus elementos. En cada estructura se observa el estado del cable conductor, los aisladores, los herrajes y el cable de guarda. El conductor y el cable de guarda son observados a lo largo de cada vano, toda esta información es recopilada, y sistematizada por cada uno de los sistemas y georreferenciada, ahora se entrena manualmente el análisis y determinación de elementos que requieran mantenimiento o el seguimiento posterior por desgaste $u$ otro fenómeno, pero 
se trabaja en crear sistemas más autónomos para la identificación, selección y análisis de los diversos elementos que son de atención para los sistemas de mantenimiento y prevención de incidentes.

\section{Objetivos}

General: Inspeccionar las áreas de interés para las líneas de transmisión mediante drone (apto para operaciones a baja altura, baja velocidad y bajo condiciones de viento moderado) dotado de los equipos de fotografía y filmación de alta resolución a color y con cámara térmica, para vanos específicos en las líneas de transmisión del alcance.

Específicos: Los servicios de inspección con el drone cubrirán por completo los conductores y los hilos de guarda, con sus respectivos anclajes y aisladores. Así como la sistematización de la información y el análisis de los datos para las respectivas sugerencias.

\section{Equipos Utilizados: DJI M300 RTK: Drone: DJI Matrice 300}

Hasta 55 minutos de vuelo

Distancia máxima 15 kilómetros

Nuevo sistema de detección y posicionamiento de 6 sensores

Pantalla principal de vuelo

Protección al agua y polvo IP45

Baliza anticolisión

Cámara FPV incorporada 960p

Tolerante a climas extremos -20 a 50 grados centígrados

Máxima resistencia a la fuerza del viento de hasta $15 \mathrm{~m} / \mathrm{s}$

Baterías intercambiables de inmediato

Inteligencia Artificial (AI)

Sistema inteligente de mantenimiento de la aeronave

Matrice 300 RTK es compatible con la base de precisión DRTK Matrice Series

Compatible con modo de vuelo dual con 2 controles remotos

Cámara con zoom (x200)

Sensor: CMOS 1/2.7", $20 \mathrm{MP}$

Objetivo DFOV: $66.6-4^{\circ}$

Distancia focal 6.83-119.94 mm (equivalente a 31.7-556.2 mm)

Apertura f/2.8-f/11 (normal), f/1.6-f/11 (escena nocturna)

\section{Enfoque $1 \mathrm{~m}$ hasta $\infty$ (panorámico), $8 \mathrm{~m}$ hasta $\infty$ (zoom)}

Modo de enfoque MF/AF-C/AF

Modo de exposición Auto/Manual

Compensación de exposición \pm 3.0 (incrementos de 1/3) 
Modo de medición: Medición puntual, medición con ponderación central

Bloqueo AE

Velocidad del obturador electrónico 1 1/8000 s

Rango ISO Video: 100-25600 Foto: 100-25600

Resolución de vídeo $3840 \times 2160$ a 30 fps, 1920×1080 a 30 fps

Formatos de vídeo MP4

Subtítulos de vídeo Compatible

Tamaño de fotografía $5184 \times 3888$

Formatos de fotografía JPEG

Cámara gran angular

Sensor CMOS 1/2.3", 12 MP

\section{Objetivo DFOV: $82.9^{\circ}$ Distancia focal: $4.5 \mathrm{~mm}$ (equivalente}

a $24 \mathrm{~mm}$ ) Apertura: $\mathrm{f} / 2.8$ Enfoque: $1 \mathrm{~m}$ a $\infty$

Modo de exposición: Auto

Compensación de exposición \pm 3.0 (incrementos de 1/3)

Modo de medición Medición puntual, medición con ponderación central

Bloqueo AE: Compatible

Velocidad de obturación: 1 1/8000 s

Rango ISO Video: 100-25600, Foto: 100-25600

Resolución de vídeo 1920×1080 a 30 fps

Formatos de vídeo MP4

Subtítulos de vídeo Compatible

Tamaño de fotografía 4056×3040

Formatos de fotografía JPEG

Serie Zenmuse Cámara térmica (Zenmuse H20T)

Sensor Microbolómetro VOx no refrigerado

\section{Objetivo DFOV: $40.6^{\circ}$ Distancia focal: $13.5 \mathrm{~mm}$ (equivalente a $58 \mathrm{~mm}$ ) Apertura: $\mathrm{f} / 1.0$ Enfoque: $5 \mathrm{~m}$ a $\infty$}

Zoom digital $1 \times 2 \times 4 \times 8 \times$

Resolución de vídeo 640×512 a $30 \mathrm{~Hz}$

Formatos de vídeo MP4

Resolución de imagen 640×512

Formato de imagen R-JPEG (16 bits)

Distancia entre píxeles $12 \mu \mathrm{m}$ 
Banda espectral 8-14 $\mu \mathrm{m}$

\section{Sensibilidad (NETD) $\leq 50 \mathrm{mK}$ a f/1.0}

Método de medición de la temperatura Medición puntual, medición de área

\section{Rango de escena De -40 a $150^{\circ} \mathrm{C}$ (alta ganancia) De -40 a $550{ }^{\circ} \mathrm{C}$ (baja ganancia)}

Alerta de temperatura Disponible

FFC Auto/Manual

Paleta White hot/Fulgurite/Iron Red/Hot Iron/Medical/Arctic/Rainbow 1/Rainbow 2/Tint/Black Hot

Telémetro láser

Longitud de onda $905 \mathrm{~nm}$

\section{Rango de medición 3-1200 m (a una superficie vertical con un diámetro de $\geq 12 \mathrm{~m}$ y una tasa de reflexión de $20 \%$ )}

Precisión de medición $\pm(0.2 \mathrm{~m}+\mathrm{D} \times 0.15 \%) \mathrm{D}$ es la distancia a una superficie vertical [1] (DJI)

\section{Metodología}

Una vez que el equipo de inspección se encuentra en la zona de interés, se procede a buscar áreas sin obstrucción vertical para definir la zona de aterrizaje y despegue. Luego se verifica que existan condiciones atmosféricas favorables antes de desplegar el equipo. Posteriormente se procede a realizar los vuelos y las grabaciones de cada tramo de conductor en los vanos de interés.

Para este tipo de inspecciones, es necesario contar con la presencia de dos operadores para el equipo. De esta forma, uno controla el movimiento del drone mientras que el otro se encarga de la grabación con las cámaras. Los pilotos realizan múltiples vuelos por vano para asegurar la calidad del material filmado.

Revisión del cable de guarda, incluyendo todos sus elementos en el punto de anclaje a la torre El cable de guarda se encuentra ubicado en la parte más elevada de la torre denominada puntita. Por esta razón el drone se posiciona sobre la torre para obtener material visual de calidad, desde allí se observan con claridad todas las partes o componentes del cable guarda, y se verifica el estado en que se encuentra. Específicamente, se hace la evaluación de los componentes, escaneo térmico y evaluación de posibles daños por descargas atmosféricas.

\section{Estado de los elementos metálicos}

Regular: El pin del aislador presenta pérdida del galvanizado, con manchas de oxidación y surgimiento de burbujas.

Malo: Surgimiento de escamas y expansión de la sección en el pin aislador.

Crítico: Cuando el pin del aislador ha experimentado una pérdida sensible del espesor, hasta el deterioro casi completo del elemento. 
Revisión de las cadenas de Aisladores

Como en el caso de la estructura metálica, para la evaluación visual de las cadenas de aisladores se emplean varias planillas dependiendo del caso particular (cadenas de suspensión sencilla y dobles y cadenas de amarre sencillas y dobles). Esta inspección consiste en observar, y tomar fotos aéreas desde ángulos donde se puedan apreciar los componentes de dichas cadenas, y verificar la condición del material aislante (porcelana o vidrio). Se verifica si están rotos o arqueados. También se verifica la presencia de corrosión en los pines, caperuza y herrajes de sujeción.

\section{Criterio para la evaluación del estado de los aisladores}

Las cadenas de aisladores en una línea de transmisión, inciden de forma directa en la operación confiable de un sistema eléctrico de potencia. Ya que deben soportar esfuerzos eléctricos y mecánicos ocasionados por descargas eléctricas, sobre tensiones, cambios bruscos de temperatura, vibraciones, etcétera.

\section{Elementos aislantes}

Regular: Cuando se observan sobre el material aislante líneas de fugas estrechas y discontinuas producto de descargas superficiales.

Malo: Cuando se observan sobre el material aislante líneas de fugas estrechas y continuas, producto de descargas superficiales.

Crítico: Existen tres criterios:

Cuando se observan sobre el material aislante líneas de fugas anchas y continuas.

En el caso de aisladores de porcelana, cuando se observa rotura o agrietamiento en la superficie aislante.

En el caso de aisladores de vidrio, cuando se observa carencia de la parte aislante.

Revisión de los conductores, separadores, amortiguadores, cuellos y conexiones terminales

El procedimiento empleado es similar al utilizado para la revisión de la cadena de aisladores; sin embargo, estos requieren la filmación del cable completo para poder identificar anomalías como filamentos sueltos, o daños físicos que perjudiquen las transmisiones.

Se requiere que el servicio se dé en dos etapas:

\section{Etapa 1:}

Inspección y levantamiento detallado de información del estado de tramos seleccionados de líneas de transmisión existentes, utilizando drones y cámaras de última tecnología. Incluye: Fotografías georreferenciadas del sitio general, fotografías y videos de extra alta resolución de los diferentes partes que componen el sistema de transmisión: conductores, conjuntos de herrajes, aisladores y otros similares, fotografías termográficas.

\section{Etapa 2:}

Estudio detallado con los resultados de la inspección y recomendaciones generales, incluyendo inspección visual integral, de vistas superiores y evaluación del estado de los conductores e hilos de guarda de las líneas de transmisión especificadas. Este estudio deberá contener como mínimo: 
- Descripción

- Inspección visual integral

- Inspección de vistas superiores

- Inspección del estado de los conductores y los respectivos hilos de guarda de las líneas de transmisión especificada, específicamente evaluación de componentes, escaneo térmico y evaluación de posibles daños por descargas atmosféricas.

\section{Entregables}

- Panel interactivo de resultados mapeados por SIG

- Resultados detallados y clasificados de la hoja de cálculo. Conjunto completo de imágenes georreferenciadas y catalogadas, la proyección a utilizar será CRTM05.

- Informe resumido del proyecto.

El contratista deberá hacer la entrega de los datos en formato digital. Deberá entregarse la información organizada por tramo de línea en carpetas. Deberá entregarse dos juegos de la información levantada.

Además, deberá entregar el estudio detallado con los resultados de la inspección y recomendaciones generales. El estudio debe contener como mínimo los siguientes puntos:

Previo a las inspecciones

- Plan preliminar del proyecto

- Visitas de evaluación de trabajo y riesgos

- Alcance detallado de los trabajos

- Cronograma de ejecución

- Currículo de los ingenieros, pilotos y técnicos que trabajarán en el proyecto

- Documentación legal, permisos y licencias requeridas para realizar el servicio en territorio nacional.

- Planes de inspecciones diarias y plan de mitigación de riesgos

Durante las inspecciones

- Equipo mínimo en sitio: ingeniero especializado en líneas de transmisión, piloto de dron y auxiliar técnico

- Drones y cámaras de última tecnología, adecuados para estudios en líneas de transmisión en operación.

- Proceso integral de recopilación de imágenes (E/S HD de extra alta resolución e Infrarrojos para escaneo térmico (IR))

- Acceso a software especializado para que el seguimiento y control del proceso de inspección en tiempo real

Posterior a las inspecciones

- Análisis exhaustivo realizado por ingenieros de vasta experiencia en la industria 
- Análisis de fotografías termográficas para identificar anomalías en conductores hilos de guarda

- Identificación de problemas de confiabilidad

\section{Resultados y discusión}

A lo largo de nuestras inspecciones comprobamos que no solo es más seguro, al no tener que escalar la torre, también requiere menos tiempo que con cualquier otro método, sin dejar de lado la gran ventaja que nos brindan los drones con su versatilidad, ya que permiten inspeccionar líneas de difícil acceso, poder acercarnos sin alterar la estabilidad del cable ni cortando la energía eléctrica. También las nuevas funcionalidades brindan la oportunidad de en ciertos segmentos realizar algunas de las operaciones de toma semiautomáticas, lo que agiliza aún más el trabajo y permite que elementos de infraestructura que pueda ser critico estas monitoreando sean realizados de manera más periódica, además la integración de otros sensores como LiDAR de nueva generación para los drones brindan la oportunidad de realizar una segunda labor a la vez que se realiza la principal de inspección.

\section{Conclusiones}

Los resultados de nuestros informes contienen información considerada necesaria para el conocimiento del personal, todos los componentes son inspeccionados en tiempo real mientras son filmados para poder tener la opinión del personal de mantenimiento que nos acompaña, todos estos datos son integrados en la bitácora para su futura inspección del material filmado, su comparación en el tiempo, el análisis y toma de decisiones respectiva y concerniente al mantenimiento de la infraestructura, la realización de estas labores de manera periódica es ya una realidad gracias a esta nueva tecnología, y además abre un mundo de posibilidades para poder integrar a estas mismas labores la posibilidad de mas datos y de mejores y mas rápidas decisiones mediante análisis de inteligencia artificial enlazada a sistemas que permitan manejar de manera expedita las respectivas ordenes de trabajo para cada caso.

\section{Referencias}

[1] DJl. "MATRICE 300 RTK." www.dji.com/, 2021, https://www.dji.com/matrice-300. Accessed 29102021.

[2] REVISTA ENERGÉTICA Vol. XXIV, No. 3/2003 Inspección integral de las líneas de transmisión.

[3] MOUBRAY, John. Reliability Centred Maintenance. Cap. 2-10. United Kingdom. 2000. 